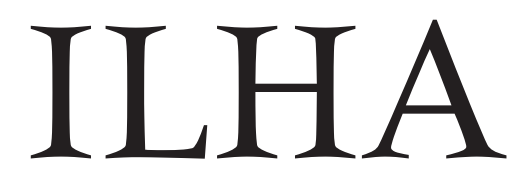

\title{
Revista de Antropologia
}

Florianópolis, volume 16, número 2

Agosto a dezembro, 2014 (2014) 
ILHA - Revista de Antropologia, publicação do Programa de Pós-Graduação em Antropologia Social (PPGAS) da Universidade Federal de Santa Catarina.

Universidade Federal de Santa Catarina

Reitora: Prof. a Roselane Neckel

Diretor do Centro de Filosofia e Ciências Humanas: Prof. Paulo Pinheiro Machado

Coordenador do PPGAS: Profa. Edviges Marta Ioris

Coordenação Editorial Maria Eugenia Dominguez, Gabriel Coutinho Barbosa e Theophilos Rifiotis

Editores do Volume Maria Eugenia Dominguez, Gabriel Coutinho Barbosa e Theophilos Rifiotis

Editora Gerente Daniela Fany Hess

Conselho Editorial Alberto Groisman, Alicia Norma Gonzalez de Castells, Antonella Maria Imperatriz Tassinari, Carmen Silvia Rial, Edviges Marta Ioris, Esther Jean Langdon, Evelyn Martina SchulerZea, Gabriel Coutinho Barbosa, Ilka Boaventura Leite, Jeremy Paul Jean LoupDeturche, José Antonio Kelly Luciani, Maria Eugenia Dominguez, Maria Regina Lisboa, Márnio Teixeira-Pinto, Miriam Hartung, Miriam Pillar Grossi, Oscar Calavia Saez, Rafael José de Menezes Bastos, Rafael Victorino Devos, Scott Correll Head, Sônia Weidner Maluf, Theophilos Rifiotis e Vânia Zikán Cardoso

Conselho Consultivo BozidarJezenik, Universidade de Liubidjana, Eslovênia; Claudia Fonseca, Universidade Federal do Rio Grande do Sul; Cristiana Bastos, Universidade de Lisboa, Portugal; David Guss, Universidade de Tufts, Estados Unidos; Fernando GiobelinaBrumana, Universidade de Cádiz, Espanha; Joanna Overing, Universidade de St. Andrews, Escócia; Manuel Gutiérrez Estévez, Universidade Complutense de Madrid, Espanha; Mariza Peirano, Universidade de Brasília; Marc-Henri Piault, Escola de Altos Estudos em Ciências Sociais, França; SoheilaShashahani, ShahidBeheshtiUniversity, Irã; Stephen Nugent, Universidade de Londres, Inglaterra

Estagiária Mariana Ribeiro dos Santos

Projeto gráfico Isabela Benfica Barbosa

Editoração eletrônica Annye Cristiny Tessaro (Lagoa Editora)

Revisão Patricia Regina da Costa (português)

Catalogação na fonte pela Biblioteca Universitária

Ilha - Revista de Antropologia / Universidade Federal de Santa Catarina. Programa de Pós-Graduação em Antropologia Social. v. 16, número 2, 2014. Florianópolis: UFSC/ PPGAS, $2014-218$ pp.

ISSN 1517-395X

1. Antropologia 2.Periódico 1. Universidade Federal de Santa Catarina

ISSN

$1517-395 X$

Solicita-se permuta/Exchange desired

As posições expressas nos textos assinados são de responsabilidade exclusiva de seus autores.

Toda correspondência deve ser dirigida à Comissão Editorial da Revista Ilha

Programa de Pós-Graduação em Antropologia, Centro de Filosofia e Ciências Humanas - CFH

Universidade Federal de Santa Catarina

Campus Universitário - Trindade

88040-970 - Florianópolis - SC - Brasil

Fone/fax: (48) 3721-9714

E-mail: ilha.revista@gmail.com sítio: http://www.periodicos.ufsc.br/index.php/ilha

Todos os direitos reservados. Nenhum extrato desta revista poderá ser reproduzido, armazenado ou transmitido sob qualquer forma ou meio, eletrônico, mecânico, por fotocópia, por gravação ou outro, sem a autorização por escrito da comissão editorial. 


\title{
SUMÁRIO
}

\author{
NÚMERO 2
}

\section{Artigos}

Philipe Descola e a Virada Ontológica na

LUIZ CÉSAR DE SÁ JÚNIOR Antropologia

Relações Alteradas - Ideias Suruwaha

MIGUEL APARICIO

sobre Animais e Caça

A Etnografia como Promessa e o "Efeito

Festa e Perfomance em Espaço Público:

PAULO RAPOSO tomar a rua!

Da "Guerra" no Complexo do Alemão à Ocupação da Favela Santa Marta: aspectos de uma política de segurança pública

Vidas de Entremeio: negros e ascensão IVO DE SANTANA social no serviço público - o caso de Salvador (BA)

\section{RESEnHAS}

GIOBELLINA BRUMANA, Fernando. 

NÚMERO 2 
